\title{
98 Otitic blast injury
}

A Is an example of stimulation damage.

$B$ Produces damage to the ear drum during the initial positive pressure wave.

C The extent of damage to the inner ear is reduced by an accompanying rupture of the ear drum.

D Only infrequently produces tinnitus.

E Does not produce vestibular symptoms.

\section{Labyrinthine window rupture}

A A preceding history of trauma can always be elicited.

$B$ The round window is more frequently affected than the oval window.

C Symptoms and audiometry may be similar to Menière's disease.

D A rupture is usually visualized under high magnification.

E Surgical closure improves the hearing in over $60 \%$ of cases.

\section{Otitic labyrinthitis}

A Is most commonly due to surgical trauma such as stapedectomy or mastoidectomy.

B May be produced by a pathological fistula.

C Can occur after an acute viral illness.

D May result in ossification of the auditory nerve.

E Can not occur by direct spread of middle ear infection via the labyrinthine window membranes since they are impermeable. 